\section{Postoperative pectoral swelling after shoulder arthroscopy}

\author{
ERSIN ERCIN, MUSTAFA GOKHAN BILGILI, HALIL NADIR ONES, CEMAL KURAL
}

Department of Orthopaedics and Traumatology, Bakirkoy Dr Sadi Konuk Training and Research Hospital, Istanbul, Turkey

\begin{abstract}
Fluid extravasation is possibly the most common complication of shoulder arthroscopy. Shoulder arthroscopy can lead to major increases in the compartment pressure of adjacent muscles and this phenomenon is significant when an infusion pump is used. This article describes a case of pectoral swelling due to fluid extravasation after shoulder arthroscopy. A 24year-old male underwent an arthroscopic Bankart repair for recurrent shoulder dislocation. The surgery was performed in the beach chair position and lasted two hours. At the end of the procedure, the patient was found to have left pectoral swelling. A chest radiography showed no abnormality. Pectoral swelling due to fluid extravasation after shoulder arthroscopy has not previously been documented.
\end{abstract}

Keywords: shoulder, arthroscopy, fluid extravasation, pectoral, swelling.

\section{Introduction}

Fluid extravasation can lead to early termination of shoulder arthroscopy due to swelling and reduced clarity of vision. More importantly it can cause serious complications such as airway compromise. Therefore it is important to make every effort to minimize this condition. Fluid extravasation is possibly the most common complication of shoulder arthroscopy.

Pectoral swelling is a rare occurrence whose causes include subpectoral abscess, pectoralis major tendon

\footnotetext{
Corresponding Author:

Ersin Ercin, MD

Department of Orthopaedics and Traumatology, Bakirkoy

Dr Sadi Konuk Training and Research Hospital

Tevfik Saglam cad. No: 11, 34147, Zuhuratbaba, Istanbul,

Turkey

E-mail: ersine@hotmail.com
}

rupture and hematoma (1). Pectoral swelling due to fluid extravasation after shoulder arthroscopy has never previously been reported. This article describes a rare case of fluid extravasation due to the use of a short cannula.

\section{Case presentation}

A 24-year-old male, weighing $95 \mathrm{~kg}$, underwent an arthroscopic Bankart repair for recurrent shoulder dislocation. The patient reported two previous shoulder dislocations and pain lasting nine months. Conservative treatment and a course of physical therapy had failed. The patient had no history of systemic disease or allergies. After discussion of the conservative and operative treatment options, the patient chose operative treatment. The preoperative diagnosis was a large labral tear. Surgery was performed under general anesthesia with the patient in the beach chair position; silicon pads were placed under his knees and head. Saline solution was used, delivered via an automatic arthroscopic pump. The pump pressure varied between 37 and $59 \mathrm{mmHg}$ during the surgery, which lasted two hours: 20 liters of irrigation fluid were used in total. At the end of the procedure, the patient was found to have left pectoral swelling (Fig. 1). A chest radiography showed no abnormality. The patient was extubated with no additional problems. The swelling resolved after six hours.

\section{Discussion}

Shoulder arthroscopy is an effective and safe procedure used to treat a wide variety of shoulder pathologies. Complications are rare, reported in between 4.6 and $10.6 \%$ of cases $(2,3)$. The major complications associated with the procedure are infection, shoulder stiffness, 


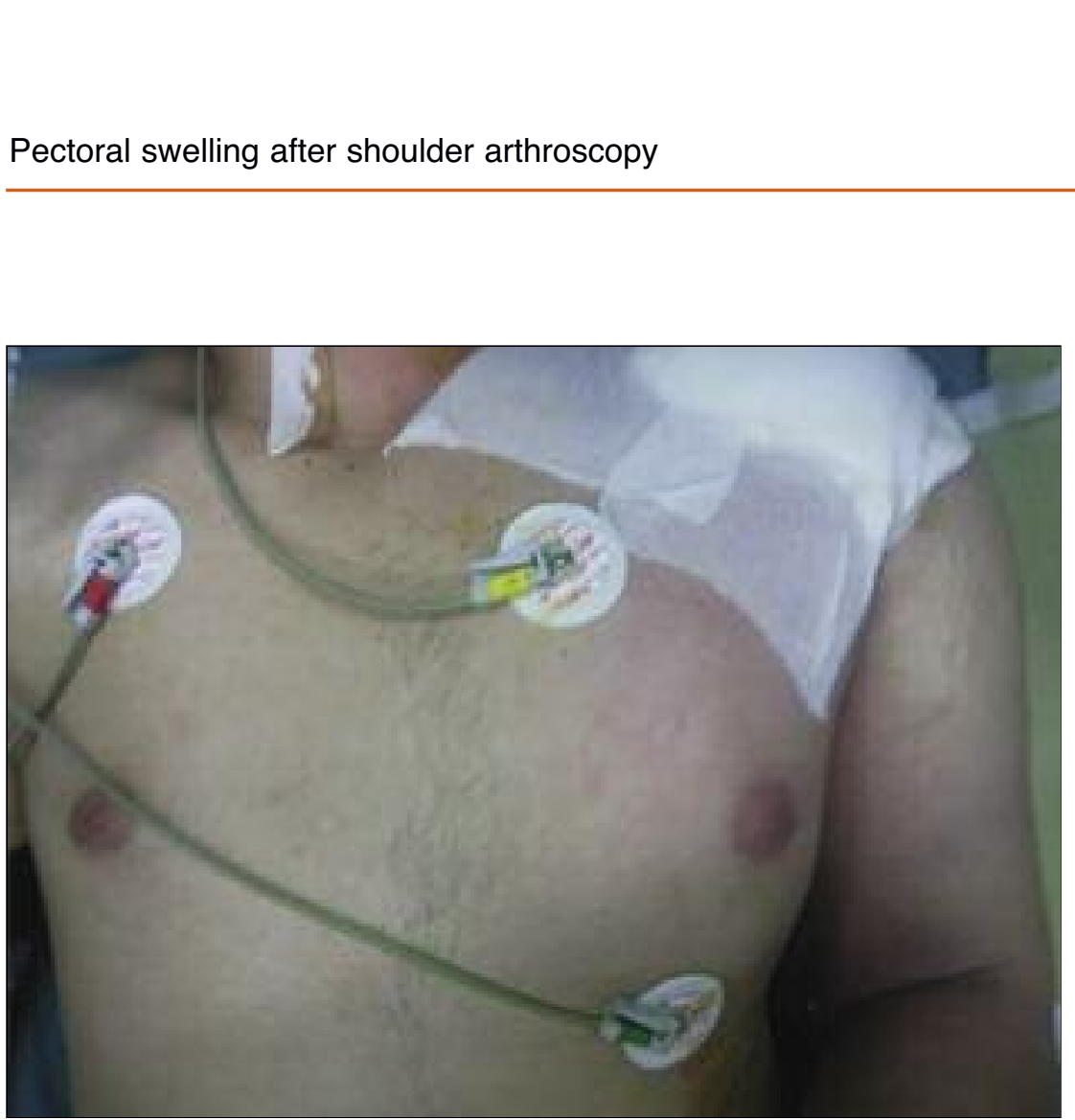

Fig. 1. Photograph of the patient with left pectoral swelling after shoulder arthroscopy.

gery, the Authors found higher pressures after surgery but stated that none of the patients had clinical problems (12).

The Authors of another study measured deltoid muscle compartment pressure and found the highest pressures during acromioplasty; they reported that compartment pressures returned to normal within four minutes of the surgery (13).

Fluid extravasation is often related to prolonged procedures, poor visualization and improper use of the infusion pump. Surgeon experience is an important factor for managing and stopping fluid extravasation. Bleeding causes poor visualization and the use of higher pressures to improve visualization can result in excessive swelling. For this reason, maintenance of homeo-

iatrogenic tendon and cartilage injury, vascular and neurological injury, reflex sympathetic dystrophy and deep venous thrombosis. Fluid retention in shoulder arthroscopy ranges from $1000 \mathrm{ml}$ to $3000 \mathrm{ml}$ (4). One of the major concerns in shoulder arthroscopy is extravasation of fluid into the surrounding soft tissues. Fluid management and awareness of fluid extravasation are very important during arthroscopic shoulder surgery. Soft-tissue extravasation can be due to the use of high pump pressures over a prolonged time and it can have serious consequences, such as upper airway obstruction (5). Also, local swelling may lead to a prolonged surgical time. Some Authors recommend marking all landmarks prior to surgery in order to avoid improper portal placement due to excessive swelling (3). Reports in the literature have outlined many complications associated with fluid extravasation. Tracheal deviation and airway compromise, negative-pressure pulmonary edema, skin necrosis, rhabdomyoloysis and neuropraxias are all reported complications of fluid extravasation during shoulder arthroscopy (2, 6-11). Shoulder arthroscopy can lead to major increases in the compartment pressure of adjacent muscles, and this phenomenon is significant when an infusion pump is used. In one study, in which deltoid and supraspinatus muscle pressures were measured before and after arthroscopic shoulder sur- stasis is very important during arthroscopic shoulder surgery. The use of hypotensive anesthesia, cold irrigation fluid and electrocautery devices, and the addition of epinephrine to the irrigation fluid are strategies that improve homeostasis $(14,15)$.

Despite the complications that have been reported, fluid extravasation is rarely problematic and usually resolves within 12 hours (13). In a prospective study of 1184 patients submitted to shoulder arthroscopy performed by 21 experienced arthroscopists, the overall complication rate was reported to be $0.7 \%$. The highest complication rate was with rotator cuff repair $(11.1 \%)$, followed by staple capsulorrhaphy (3.3\%) and anterior acromioplasty (1.1\%) (16). Correct portal placement and the use of longer cannulas can reduce the risk of fluid extravasation. With regional anesthesia, less postoperative analgesia is needed and the hospital stay is shorter. Also, blood pressure adjustments are easier in regional anesthesia. Control of bleeding is also important; this can be achieved by proper use of electrocautery devices and by the use of local vasoconstrictors. We recommend that infusion pumps be used for the shortest time possible or that gravity inflow be used, as desired. A separate portal for inflow is hard to control and its use may cause extravasation to the surrounding tissues. Inflow 


\section{Toints}

through the arthroscopic sheath may be a better choice to prevent this extravasation. Constant monitoring of pumps and pump pressure is critical during shoulder arthroscopy.

In conclusion, early recognition of increased swelling during surgery, careful positioning of portals, and the use of appropriate equipment to reduce surgical times are all crucial in minimizing complications due to fluid extravasation in shoulder arthroscopy. Careful postoperative monitoring is also critical.

\section{References}

1. Tsai HB, Lin CW, Kuo CC, Huang JW, Hung KY. An 86-yearold man with a unilateral pectoral swelling. Neth Med J. 2010; 68:251.

2. Brislin KJ, Field LD, Savoie FH 3rd. Complications after arthroscopic rotator cuff repair. Arthroscopy. 2007;23:124128.

3. Berjano P, González BG, Olmedo JF, Perez-España LA, Munilla MG. Complications in arthroscopic shoulder surgery. Arthroscopy. 1998;14:785-788.

4. Lo IK, Burkhart SS. Immediate postoperative fluid retention and weight gain after shoulder arthroscopy. Arthroscopy. 2005;21:605-610.

5. Borgeat A, Bird P, Ekatodramis G, Dumont C. Tracheal compression caused by periarticular fluid accumulation: a rare complication of shoulder surgery. J Shoulder Elbow Surg. 2000;9:443-445.

\section{E. Ercin et al.}

6. Weber SC, Abrams JS, Nottage WM. Complications associated with arthroscopic shoulder surgery. Arthroscopy. 2002;18 (2 Suppl 1): 88-95.

7. Jirativanont T, Tritrakarn TD. Upper airway obstruction following arthroscopic rotator cuff repair due to excess irrigation fluid. Anaesth Intensive Care. 2010;38:957-958.

8. Gogia AR, Bajaj J, Sahni A, Saigal D. Negative-pressure pulmonary oedema in a patient undergoing shoulder arthroscopy. Indian J Anaesth. 2012; 56:62-65.

9. Pitman MI, Nainzadeh N, Ergas E, Springer S. The use of somatosensory evoked potentials for detection of neuropraxia during shoulder arthroscopy. Arthroscopy. 1988;4:250255.

10. Mohammed KD, Hayes MG, Saies AD. Unusual complications of shoulder arthroscopy. J Shoulder Elbow Surg. 2000; 9:350-353.

11. Lim JK, Ang KC, Wang SC, Kumar VP. Rhabdomyolysis following shoulder arthroscopy. Arthroscopy. 2006;22:1366.

12. Carr CF, Murphy JM. Deltoid and supraspinatus muscle pressures following various arthroscopic shoulder procedures. Arthroscopy. 1995;11:401-403.

13. Ogilvie-Harris D, Boynton E. Arthroscopic acromioplasty: Extravasation of fluid into the deltoid muscle. Arthroscopy. 1990;6:52-54.

14. Morrison DS, Schaefer RK, Friedman RL. The relationship between subacromial space pressure, blood pressure, and visual clarity during arthroscopic subacromial decompression. Arthroscopy. 1995;11:557-560.

15. Jensen KH, Werther K, Stryger V, et al. Arthroscopic shoulder surgery with epinephrine saline irrigation Arthroscopy. 2001;17: 578-581.

16. Small NC. Complications in arthroscopic surgery performed by experienced arthroscopists. Arthroscopy. 1998;4:215-221. 